\title{
Reacción adversa a medicamentos como causa de hospitalización de emergencia de adultos mayores
}

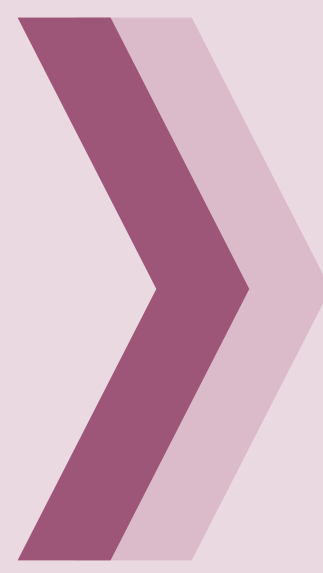

\author{
Adverse drug reactions in \\ elderly patients as reason of \\ emergency hospitalization
}

José Amado-Tineo, ${ }^{1}$ Rolando Vásquez-Alva, ${ }^{1}$ César Rojas-Moya, ${ }^{1}$ Teodoro Oscanoa-Espinoza ${ }^{2}$

1. Médico internista Hospital E. Rebagliati, Essalud. Lima, Perú. Magíster en Docencia e Investigación en Salud.

2. Médico geriatra, Hospital G. Almenara, Essalud. Lima, Perú. Doctor en Medicina.

\section{RESUMEN}

INTRODUCCIÓN. Las reacciones adversas a medicamentos son frecuentes y prevenibles. Овлетіvos. Describir la medicación habitual, potencialmente inadecuada y reacciones adversas como causa de hospitalización. Material Y métodos. Estudio descriptivo en hospitalizados de 65 años o más, en emergencia del hospital Rebagliati. Muestreo sistemático de 238 pacientes. Entrevista a paciente o cuidador y revisión de historia clínica. Instrumentos: criterios STOPP para prescripción inadecuada y algoritmo de Karl y Lasagna para reacciones adversas. Resultados. Masculino 47,1\%, edad promedio 78,36 $( \pm 7,83)$ años; 238 pacientes recibian 731 fármacos (en promedio, 3 fármacos por paciente), los más frecuentes fueron enalapril y ácido acetilsalicílico. Medicación potencialmente inadecuada en $24,6 \%$, mayor frecuencia glibenclamida y digoxina. De los ingresos, 7,6 \% tenian como causa probable reacción adversa a medicamentos; los más frecuentes fueron glibenclamida, insulina y clopidogrel y su presentación clínica más frecuente, hipoglicemia, arritmia cardíaca y sangrado digestivo. Conclusiones. La medicación habitual más frecuente fue cardiovascular, alta frecuencia de medicación potencialmente inadecuada y reacción adversa a medicamentos como causa de ingreso.

Palabras claves: reacción adversa a medicamentos; prescripción inadecuada; adulto mayor; hospitalización en emergencia.

\begin{abstract}
INTRODUCTION. Adverse drug reactions are frequent and avoidable. Aıms. To describe habitual medication, potentially inadequate medication and adverse drug reactions as reason of hospitalization. MATERIAL AND METHODS. Descriptive study in 65 or more yearold inpatients, emergency of Rebagliati hospital. Systematic sampling of 238 patients. Interview to patient or keeper and checked clinical history. Instruments: Screening Tool of Older Person's Potentially Inappropriate and Karl and Lasagna's algorithm. Results. Male 47,1\%, average age 78,36 $( \pm 7,83)$ years. 731 medicaments were prescribed in 238 patients ( 3 medicaments for patient), enalapril and aspirin were most frequent. Potentially inadequate medication was in $24,6 \%$, being the most frequent glibenclamide and digoxin. 7,6\% of inpatient presented adverse drug reaction as reason of admission; glibenclamide, insulin and clopidogrel were frequent; and the clinical presentation was hypoglycemia, arrhythmia and digestive bleeding. Conclusions. The frequent habitual medication was cardiac-vascular, frequent potentially inadequate medication and adverse drug reactions patients as reason of hospitalization.
\end{abstract}

KEY WORDS: adverse drug reaction, inappropriate prescribing; elderly patient; emergency hospitalization. 


\section{INTRODUCCIÓN}

En las últimas décadas, las mejoras económicas y en salud de América Latina y el mundo han disminuido la tasa de mortalidad y aumentado la expectativa de vida, con un aumento significativo de la población adulta mayor. En Perú, según datos de 2007, los mayores de 65 años constituyen $6,4 \%$ de la población nacional, con tasa de crecimiento anual de 3,9\% (la más alta de los grupos etarios). En Lima y Callao, residen $36 \%$ de esta población. ${ }^{1-3}$

Las personas adultas mayores son un grupo heterogéneo vulnerable, debido a su elevada tasa de morbilidad crónica y discapacidad, por lo que requieren especial atención sociosanitaria. En Lima, la demanda de atención en salud, más frecuente y recurrente cada día, rebasa la capacidad de atención de las principales instituciones prestadoras de salud, con el consecuente aumento del sufrimiento del paciente y su familia. Este problema es mayor en los servicios de urgencias. ${ }^{1}$

En 2007, 69,4\% de los pacientes hospitalizados en un servicio de Medicina Interna del hospital Rebagliati (Lima, Perú) eran mayores de 60 años y con alta frecuencia de polifarmacia. Las múltiples enfermedades 0 factores de riesgo presentes en este grupo etario obligan a emplear varios medicamentos por periodos prolongados, los cuales presentan problemas relacionados a medicamentos (sobreprescripción, disprescripción, subuso, reacción adversa-RA-y adherencia), lo que incrementa la complejidad de estos pacientes. ${ }^{2,45}$

Se han definido listas de medicamentos con los cuales el riesgo de sufrir efectos adversos es superior al beneficio clínico, en especial cuando existen alternativas terapéuticas más seguras $y / 0$ eficaces. La prescripción inapropiada también incluye el uso de fármacos con una mayor frecuencia o mayor duración de la indicada, el uso de fármacos con un elevado riesgo de interacciones medicamento-medicamento 0 medicamentoenfermedad y fármacos duplicados o de la misma clase. La prescripción inapropiada incluye, además conceptualmente, la no utilización de fármacos beneficiosos que sí están clínicamente indicados, pero que a menudo no se prescriben en pacientes mayores por razones no bien determinadas. ${ }^{2,6-8}$

La forma más extendida de evaluar esta calidad de prescripción es con indicadores o criterios explícitos desarrollados con técnicas de consenso, opinión de expertos o revisión extensa de la bibliografía, orientada básicamente en el fármaco y/o enfermedad o condición del paciente. Estos indicadores explícitos tienen la ventaja de brindar la posibilidad de realizar estudios farmacoepidemiológicos, que tienen como fuente de datos las historias clínicas y los servicios farmacéuticos, y son útiles en evaluaciones globales y en grandes poblaciones. ${ }^{2,8}$

Entre los criterios explícitos más conocidos están los de Beers (2007) y los STOPP/START (Screening Tool of Older Person's Potentially inappropriate / Screening Tool to Alert doctors to the Right indicate Treatment) (2008). Los criterios de Beers fueron realizados en $E E U U$, identifican los 58 fármacos 0 clases de fármacos y especifica sus potenciales RA y señalan 20 enfermedades o condiciones en las cuales ciertos medicamentos no deberían ser usados. Los criterios STOPP/START fueron desarrollados en Irlanda y en Reino Unido, su acrónimo describen los objetivos fundamentales de la prescripción adecuada de medicamentos en el paciente adulto mayor (PAM), suspender los fármacos inadecuados y evitar la infraprescripción de otros. ${ }^{2,6,8}$

El empleo de medicamentos en el PAM constituye cerca de $30 \%$ del gasto en salud. El gasto estimado en EE UU por uso inapropiado de medicamentos es 20 billones de dólares anuales. Factores asociados son la pobre situación económica, la polifarmacia, la edad mayor de 85 años, el uso de sedantes, el vivir solo y la depresión. ${ }^{9,10}$

Según reportes internacionales, la frecuencia de prescripción inapropiada de fármacos en PAM varía de $14 \%$ a $37 \%$ en pacientes institucionalizados, hasta $40 \%$ en residentes de hogares de reposo; con mayor frecuencia de efectos adversos y causa de $10 \%$ a $30 \%$ de ingresos hospitalarios, con pérdidas económicas muy importantes. Así, produce un gran impacto en la salud pública, el cual está en incremento y en muchos casos es inaceptable., ${ }^{911-21}$

Por tal motivo, se plantearon como objetivos describir la medicación habitual, determinar medicación potencialmente inadecuada e identificar RA a medicamentos (RAM) como causa de hospitalización en PAM atendidos en emergencia del Hospital Nacional Edgardo Rebagliati Martins (HNERM), EsSalud, durante el año 2011. 


\section{MATERIAL Y MÉTODOS}

Estudio descriptivo de corte transversal, mediante la evaluación de la medicación habitual, la medicación potencialmente inadecuada, las RAM y las variables sociodemográficas del paciente.

La población estuvo constituida por pacientes de 65 años o más hospitalizados por causas médicas en el servicio de emergencia del HNERM, EsSalud, ubicado en el distrito de Jesús María, en Lima, Perú, durante el año 2011.

El tamaño de muestra (238 pacientes) se calculó según la fórmula de proporciones. Se consideró un margen de error de $0,06, p=25 \%$, según frecuencia de polifarmacia de un estudio previo del mismo hospital; 22000 ingresos anuales a salas de observación el año previo y un ajuste por pérdidas de $10 \%$.

Se empleó un muestreo sistemático. Del libro de reporte diario de las salas de emergencia, se seleccionó los ingresos cada cinco días, durante el período enero a junio de 2011. Se evaluaron 255 pacientes, se excluyeron 5 por negarse a participar en el estudio y 12 por datos incompletos.

En los pacientes seleccionados, se revisó la historia clínica de emergencia y la historia clínica antigua y se entrevistó al paciente o a su cuidador, si el paciente no brindaba datos confiables. Los datos fueron recolectados por cuatro médicos internistas del servicio de emergencia.

Se evaluó la medicación potencialmente inadecuada, según los criterios STOPP, preguntando al paciente 0 al cuidador la medicación habitual consumida durante los 15 días o más previos a la hospitalización, lo que se complementó con lo registrado en la historia clínica antigua.

Se consideró RAM a la que cumplía los criterios de relación causal probable según el algoritmo de Karl y Lasagna (secuencia temporal, respuesta conocida al fármaco, ausencia de explicación alternativa para la reacción y mejoría al retirar el medicamento). ${ }^{22}$

Se revisó el correcto llenado de las fichas de recolección de datos y se codificó y procesó los datos con el programa estadístico SPSS 17.00. Se describen datos empleando medidas de distribución de frecuencia y tendencia central; frecuencias y porcentajes para las variables discretas y media con desviación estándar para las variables continuas.

El proyecto fue aprobado por la Oficina de Capacitación, Docencia e Investigación de la Red Asistencial Rebagliati-EsSalud. Se solicitó consentimiento informado al paciente 0 al cuidador. Los pacientes fueron evaluados siguiendo los principios de respeto y de confidencialidad y los criterios de buenas prácticas clínicas. Se notificó al médico tratante en los casos detectados de medicación potencialmente inadecuada, y se sugirió el retiro o el cambio de la medicación involucrada.

\section{RESULTADOS}

De los pacientes evaluados, $47,1 \%$ fueron de sexo masculino, la edad varió entre 65 y 101 años, con un promedio de 78,36 $( \pm 7,83)$ años. Los diagnósticos más frecuentes fueron hipertensión arterial (56\%), diabetes mellitus tipo 2 (25\%), falla renal crónica (12\%), neumonía (12\%) e infección urinaria (12\%).

Los 238 pacientes recibían 731 fármacos, que correspondieron a 113 principios activos de medicamentos y a 91 subgrupos según la clasificación ATC (anatómica, terapéutica y química) de fármacos. En promedio, cada paciente recibió 3 fármacos, con rango 0-10.

La Tabla 1 presenta los principios activos y la Tabla 2, el subgrupo químico de medicamentos más frecuentemente utilizados como medicación

Tabla 1. Medicamentos más utilizados en adultos mayores hospitalizados en emergencia del HNERMEsSalud 2011

$\begin{array}{lcc}\text { Medicamentos } & \mathrm{n} & \% \\ \Delta \text { Enalapril } & 59 & 24,8 \\ \Delta \text { Ácido acetil salicílico } & 43 & 18,1 \\ \Delta \text { Furosemida } & 30 & 12,6 \\ \Delta \text { Captopril } & 30 & 12,6 \\ \Delta \text { Alprazolam } & 26 & 10,9 \\ \Delta \text { Omeprazol } & 26 & 10,9 \\ \Delta \text { Ácido fólico } & 21 & 8,8 \\ \Delta \text { Metformina } & 21 & 8,8 \\ \Delta \text { Ranitidina } & 21 & 8,8 \\ \Delta \text { Amlodipino } & 20 & 8,4\end{array}$


Tabla 2. Subgrupo químico de medicamentos más utilizados en adultos mayores hospitalizados en emergencia del HNERM-EsSalud, 2011

\begin{tabular}{|c|c|c|}
\hline Subgrupo químico de medicamentos & $\mathrm{n}$ & $\%$ \\
\hline$\triangle \mathrm{IECA}$ & 90 & 37,8 \\
\hline$\Delta$ Diuréticos de techo alto-sulfonamidas & 43 & 18,1 \\
\hline$\Delta$ Ácido acetilsalicílico y derivados & 43 & 18,1 \\
\hline A Calcioantagonistas dihidropiridinas & 31 & 13,0 \\
\hline$\Delta$ Benzodiacepinas & 28 & 11,8 \\
\hline$\Delta$ Inhibidores bomba de protones & 26 & 10,9 \\
\hline$\triangle \mathrm{ARA} \|$ & 26 & 10,9 \\
\hline$\Delta$ B-bloq selectivos & 23 & 9,7 \\
\hline$\triangle$ Antagonistas $\mathrm{H} 2$ & 21 & 8,8 \\
\hline$\Delta$ Biguanidas & 21 & 8,8 \\
\hline$\Delta$ Sulfonilureas & 20 & 8,4 \\
\hline
\end{tabular}

habitual. Se detectó medicación potencialmente inadecuada en 58 pacientes $(24,6 \%)$, su detalle se muestra en la Tabla 3 y los grupos identificados según la clasificación ATC, en la Figura 1.

De los pacientes evaluados, 7,6\% tenían como causa probable de ingreso una RAM, estos tenían una edad promedio de 77 años (rango 65-96)

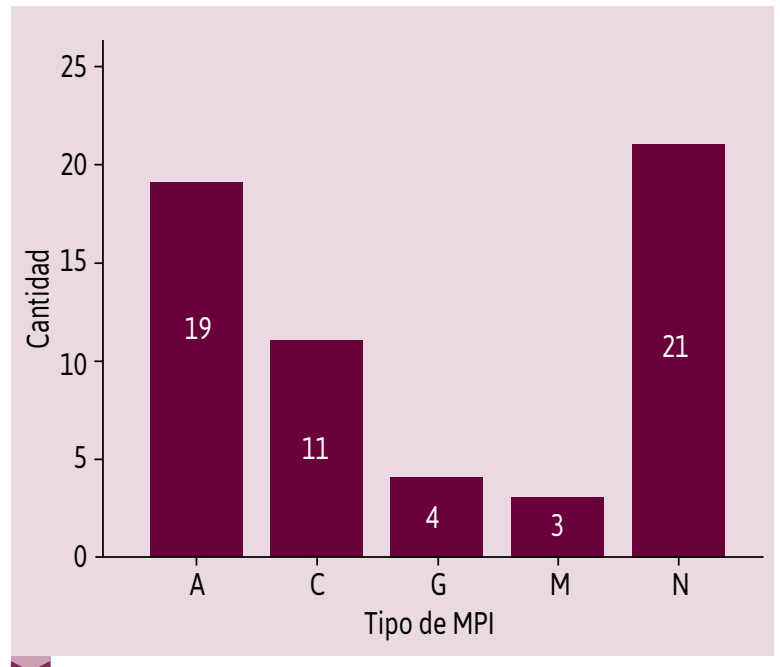

Figura 1. Medicación potencialmente inadecuada (MPI), en clases de fármacos según clasificación anatómica-química (ATC) en adultos mayores hospitalizados en emergencia del HNERM-EsSalud 2011.

A: tracto alimentario y del metabolismo; C: sistema cardiovascular; $G$ : sistema genitourinario $u$ hormonas sexuales; $M$ : sistema musculo esquelético; $\mathrm{N}$ : sistema nervioso.

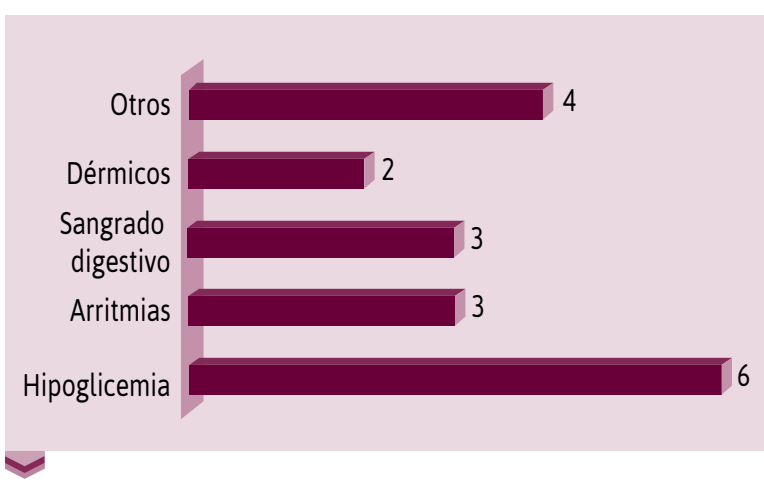

Figura 2. Manifestaciones clínicas de reacciones adversas a medicamentos en adultos mayores hospitalizados en emergencia HNERM-EsSalud 2011.

y edad mayor o igual de 85 años en 22,2\%; $50 \%$ eran de sexo masculino; $72 \%$ usaban de tres a cinco medicamentos y $38 \%$ tenían medicación potencialmente inadecuada.

La presentación clínica de las RAM se muestra en la Figura 2. Las arritmias correspondieron a fibrilación auricular, dos casos con respuesta ventricular baja y uno con respuesta alta; los casos de sangrados digestivos fueron altos y los cuadros dérmicos, urticaria; en otros se incluye un caso de síndrome confusional y otro de falla cardíaca. Los medicamentos relacionados a estas RA se muestran en la Figura 3, se incluye en otros a diltiazem, gabapentina, levodopa y verapamilo. Todos los pacientes tuvieron evolución favorable.

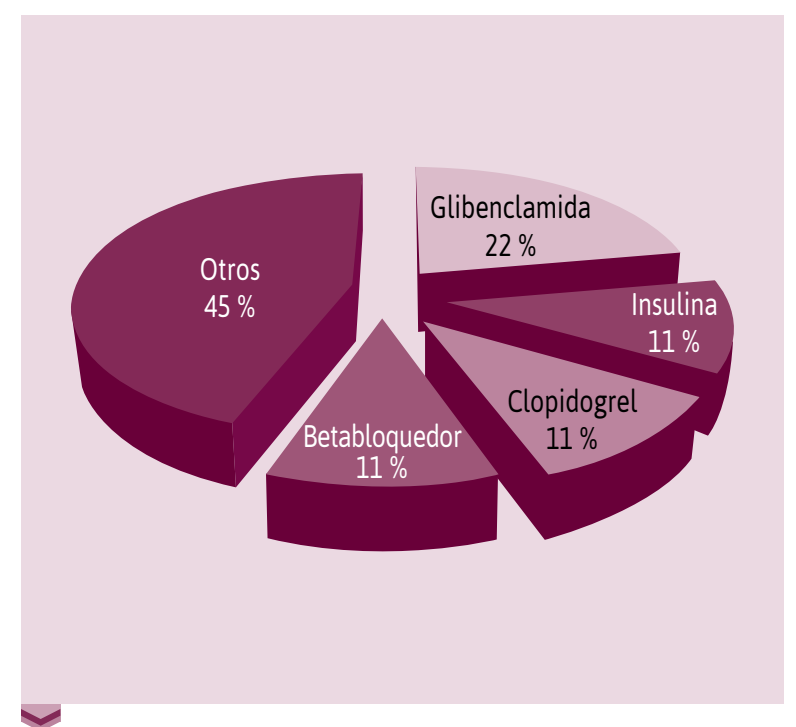

Figura 3. Medicamentos relacionados a reacciones adversas en adultos mayores hospitalizados en emergencia del HNERM-EsSalud 2011. 
Tabla 3. Medicamentos potencialmente inadecuados según criterios STOPP en pacientes adultos mayores hospitalizados en emergencia HNERM-EsSalud, 2011

Medicamento $\triangle$ Glibenclamida

n

12

$\Delta$ Digoxina

114,6

$\triangle$ Nifedipino $10 \mathrm{mg}$

$\triangle$ Diazepam

$\triangle$ Risperidona

- Terazocina

$\triangle$ Amitriptilina

$\triangle$ Biperideno

$\triangle$ Nimodipino

$\Delta$ Ibuprofeno
5,0

\section{$\%$}

2,1

1,7

1,7

1,3

1,3

1,3

0,8

\section{DISCUSIÓN}

El elevado número de diagnósticos de ingreso a emergencia se relacionan con la elevada frecuencia de pluripatología de este grupo de pacientes. La hipertensión arterial, al igual que en Cuba es la más frecuente, seguida, a diferencia de Cuba, de diabetes mellitus, falla renal crónica y cirrosis hepática; con baja frecuencia de osteoartrosis y cardiopatía crónica. ${ }^{17}$

Se identificó un amplio número de medicamentos utilizados por los pacientes, con un promedio de tres fármacos por paciente. Este promedio es menor a otros estudios locales e internacionales, probablemente por subregistro, pero igual a lo reportado en Cuba en pacientes ambulatorios.,17,18

Los medicamentos más utilizados fueron los inhibidores dela enzima convertidora deangiotensina (IECA) captopril y enalapril, el ácido acetilsalicílico, a dosis antiagregante plaquetaria, y la furosemida. El uso del ácido acetilsalicílico, similar a otros estudios, se podría explicar por la alta frecuencia del antecedente de enfermedad cerebrovascular. El uso crónico de furosemida podría desencadenar efectos adversos..$^{14} \mathrm{~A}$ diferencia de otros reportes, son bajas la frecuencia de analgésicos, probablemente por omisión y subregistro, y de insulina y warfarina, por temor del paciente o del médico prescriptor. ${ }^{14,17}$

El uso de omeprazol tiene mayor frecuencia en los pacientes que no presentaron síndrome confusional y podría no ser considerada medicación potencialmente inadecuada porque no se obtuvo el tiempo de uso de este medicamento.

El empleo de criterios STOPP es el más práctico y actualizado, con un mayor porcentaje de identificación de medicamentos potencialmente inadecuada (MPI) que los criterios de Beers, pero menos que los criterios STARTT. Este último no se aplicó por falta de data confiable. ${ }^{18,19}$

La frecuencia de MPI $(24,6 \%)$ fue similar a lo reportado en Chile ${ }^{20}$ y ligeramente menor a lo reportado por Oscanoa en $2005(29,4 \%)^{11}$ y en 2011.7 Un reporte de Bogotá, Colombia, presenta una tasa más baja de $\operatorname{MPI}(21,5 \%)$, pero en PAM ambulatorios. ${ }^{12}$

Entre los MPI más usados se encontró la glibenclamida, la digoxina, a dosis mayor a 125 $\mathrm{mg} / \mathrm{d}$, nifedipino de acción corta, benzodiacepinas de acción prolongada (diazepam), antipsicóticos (risperidona, amitriptilina) y terazocina.

Los fármacos identificados como potencialmente inadecuados fueron similares a los reportados por Oscanoa, a excepción de piroxicam, que actualmente no se encuentra en el petitorio de Essalud. En el presente estudio aparece la glibenclamida como el más frecuente, la que recién en los últimos años se clasificó como MPI.?

Existe una alta frecuencia de uso de antidiabéticos orales similar a lo reportado en EE UU. El grupo de fármacos potencialmente inadecuados según ATC más frecuentes fueron los del sistema nervioso (benzodiacepinas) y del tracto alimentario (sulfonilureas). Difiere del estudio de Colombia por la baja frecuencia de relajantes musculares, antihistamínicos anticolinérgicos, y la asociación anticoagulantes más ácido acetilsalicílico. ${ }^{14,20}$

En EE UU, se reporta mayor riesgo de alucinacionesdelirio en los pacientes que recibieron narcóticos, pero no en los que recibieron anticolinérgicos, sin embargo, no se encuentra esta relación en este trabajo, por la baja frecuencia de uso de estos medicamentos. ${ }^{9}$

Similar a lo reportado en EE UU, los antidiabéticos y antiagregantes plaquetarios fueron los fármacos más relacionados con los efectos adversos que requirieron hospitalización, pero se evidencia la ausencia importante de warfarina por un subuso en la población en estudio. También se destaca la 
baja presencia de otros medicamentos de las listas explícitas de riesgo, probablemente porque no se evaluaron efectos adversos menores. ${ }^{14}$

Como principales limitaciones del presente estudio, se tiene que los pacientes estudiados son exclusivamente beneficiarios de un sistema de salud público, no incluye población de menores recursos económicos. En cuanto a la evaluación de la medicación habitual, no se incluyó el tiempo de tratamiento ni la adherencia al tratamiento.

Se encontró importante subregistro de medicación habitual en la historia clínica hospitalaria (antigua y de ingreso). Existe también escaso interés del personal de salud en el tema, debido quizá al exceso de trabajo diario o la falta de información al respecto.

\section{CONCLUSIONES}

Enalapril, ácido acetilsalicílico, furosemida y captopril son los medicamentos más usados como medicación habitual en adultos mayores internados en emergencia. Cada paciente recibe un promedio de tres fármacos. La medicación potencialmente inadecuada, según criterios STOPP, es frecuente en pacientes internados en emergencia. El grupo más frecuente de medicamentos es el del sistema nervioso y los principios activos más frecuentes, glibenclamida, digoxina, nifedipino de acción corta y diazepam. Entre los adultos mayores hospitalizados en emergencia, 7,6\% tienen como causa probable una reacción adversa a medicamentos. Los antidiabéticos (glibenclamida e insulina) y los antiagregantes plaquetarios (clopidogrel) son los más relacionados.

\section{REFERENCIAS BIBLIOGRÁFICAS}

I. Varela L, Chávez H, Herrera A, Méndez F, Gálvez M. Perfil del adulto mayor Perú-INTRA II 2004. Desarrollando respuestas integradas de los sistemas de cuidados de salud para una población en rápido envejecimiento. Lima: OPS-OMS; 2004. [citado dic 20I I]. Disponible en: http://www.minsa.gob.pe/portal/Servicios/SuSaludEsPrimero/ AdultoMayor/INFORME\%20PERFIL\%20ADULTO\%20MAYORFINAL\%20A4.doc

2. Oscanoa T, Castañeda B. Calidad de la prescripción farmacológica en geriatría. Instrumentos de evaluación, 2. ${ }^{\text {Ed }}$. Lima: Concytec; 2012.

3. INEl. Perfil Sociodemográfico del Perú. Censos Nacionales 2007: XI de población y VI de vivienda, 2. ${ }^{\text {a }}$ edición. Lima: INEl; 2008.

4. Rojas DV. Morbilidad y mortalidad del adulto mayor en un servicio de medicina de un hospital general del Perú. Rev Per Epidemiol. 2010;14(2):99-107.

5. Amado J, Chucas L, Rojas C,Pintado S, et al.Factores asociados a síndrome confusional agudo en adultos mayores internados en emergencia de un hospital terciario.An Fac Med. 2013;74:193-197.

6. Delgado E, Muñoz M, Montero B, Sánchez et al. Prescripción inapropiada de medicamentos en los pacientes mayores: los criterios STOPP/START. Rev Esp Geriatr Gerontol. 2009;44(5):273-279.

7. Oscanoa TJ. Diagnóstico de problemas relacionados con medicamentos en adultos mayores al momento de ser hospitalizados. Rev Peru Med Exp Salud Publica. 201 I;28:256-263.

8. Oscanoa TJ. Farmacología clínica en geriatría. Lima: Editorial y gráfica Ebra EIRL; 2006.

9. Stockl KM, Le L, Zhang S, Harada ASM. Clinical and economic outcomes associated with potentially innappropriate prescribing in the elderly. Am J Manag Care. 2010;16(I):el-el0.

10. Gallagher P, Barry P, O'Mahony D. Innappropriate prescribing in the elderly. I Clin Pharm Therap. 2007;32(2): I|3-121.

II. Oscanoa TJ. Prescripción potencialmente inadecuada de medicamentos en adultos mayores. [Tesis doctoral]. Lima: UNMSM; 2005.

12. Holguín-Hernández E, Orozco-Díaz G. Medicación potencialmente inapropiada en ancianos de un hospital de primer nivel, Bogotá 2007.

\section{Correspondencia}

Dr. José Percy Amado-Tineo: jpamadot@hotmail.com

Fecha de recepción: 3 de octubre de 2014

Fecha de aceptación: 6 de octubre de 2014
Rev Salud Publica. 2010; 12(2): 287-299.

13. Fiss T, Dreier A, Meinke C, et al. Frequency of inappropriate drugs in primary care: analysis of a simple of immobile patients who recieved periodic home visits. Age Ageing. 201 I;40:66-73.

14. Budnitz DS, Lovegrove MC, Shehab N, Richards CL. Emergency hospitalizations for adverse drug events in older Americans. $\mathrm{N}$ Engl J Med. 20I I;365(2I):2002-20I2.

15. Wu TY, Jen MH, Bottle A, Molokhias M, Aylin P, Bell D, et al. Ten-years trends in hospital admissions for adverse drug reactions in England 1999. 2009.J R Soc Med. 2010; 103:239-250.

16. Hamilton HJ, Gallagher PF, O'Mahony D. Inappropriate prescribing and adverse drug events in older people. BMC Geriatrics. 2009; 9:5.

17. Martínez C, PérezVT, Carballo M, Larrondo J. Polifarmacia en los adultos mayores. Rev Cubana Med Gen Integr. 2005;2I(I-2).

18. Ryan C, O'Mahony D, Kennedy J, Weedle P, Byrne S. Potentially inappropriate prescribing in Irish elderly population in primary care. $\mathrm{Br}$. Clin Pharmacol. 2009; 68(6):936-947.

19. Pattanaworasate W, Emmerton L, Pulver L, Winckel K. Comparison of prescribing criteria in hospitalised Australian elderly. Pharm Pract (Granada). 2010;8(2):132-138.

20. Jirón M, Escobar L, Orellana S, Jara P, Oyarzún X, et al. Prescripción de medicamentos potencialmente inapropiados en adultos mayores hospitalizados. In: Proceedings of the ISPOR 2nd Latin America Conference; 2009 Sep 10-I2; Rio de Janeiro [citado dic 20I I]. Disponible en: http://www.ispor.org/awards/2ndLAC/CO2.pdf

21. Berdot S, Bertrand M, Dartigues JF, Fourrier A, Tavernier B, Ritchie K, et al. Inappropriate medication use and risk of falls $-A$ prospective study in large community-dwelling elderly cohort. BMC Geriatr. 2009; 9:30.

22. Armijo JA, González M. Estudios de seguridad de medicamentos: Métodos para detectar las reacciones adversas y Valoración de la relación causa-efecto. Estudio de seguridad de medicamentos. Ensayo clínico de España. Madrid: Farmindustria; 2000. p. 162-190. [citado ene 2012]. Disponible en: http://www.farmaindustria.es/idc/groups/public/ documents/publicaciones/farma 1031.pdf\#page=|53

\section{Conflictos de interés}

El autor declara no tener conflictos de interés durante el planteamiento, ejecución de la investigación y la elaboración del artículo para su publicación. 